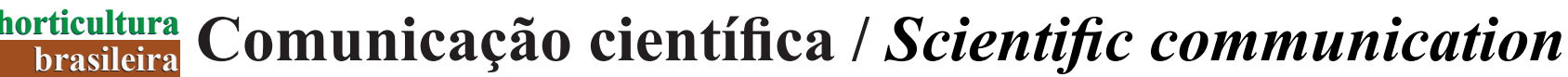

AZEVEDO AM; ANDRADE JÚNIOR VC; FERNANDES JSC; PEDROSA CE; OLIVEIRA CM. 2015. Desempenho agronômico e parâmetros genéticos em genótipos de batata-doce. Horticultura Brasileira 33: 084-090. DOI - http://dx.doi.org/10.1590/S0102-053620150000100014

\section{Desempenho agronômico e parâmetros genéticos em genótipos de batata-doce}

\begin{abstract}
Alcinei M Azevedo1; Valter C Andrade Júnior²; José SC Fernandes²; Carlos E Pedrosa ${ }^{3}$; Celso M Oliveira ${ }^{3}$ ${ }^{1}$ UFV, 36570-000 Viçosa-MG; alcineimistico@hotmail.com; 2UFVJM, C. Postal 3037, 39100-000 Diamantina-MG; valterjr@ufvjm.edu. br; cunha.fernandes@ufvjm.edu.br; ${ }^{3} U F L A$, C. Postal 3037,37200-000 Lavras-MG; carlosenrrik@yahoo.com.br; celsodoliveira@yahoo. com.br
\end{abstract}

\section{RESUMO}

O presente trabalho teve por objetivo avaliar o desempenho agronômico em genótipos de batata-doce e estimar parâmetros genéticos. O experimento foi composto por 65 variedades (clones do banco de germoplasma de batata-doce da Universidade Federal dos Vales do Jequitinhonha e Mucuri), instalado num delineamento em blocos ao acaso com três repetições e 15 plantas por parcela. Foram avaliadas a produtividade de massa verde das ramas (MV), produtividade total das raízes (PT), peso médio das raízes totais (PMT), produtividade de raízes comerciais (PC), peso médio das raízes comerciais (PMC), formato das raízes (FORM) e resistência a insetos (RI). Considerando-se os ganhos de seleção estimados para estas características, infere-se que a seleção de clones pode levar a progressos genéticos significativos. Os caracteres PT, PMT, PC, e PMC estão positivamente correlacionados, logo, a seleção em qualquer um deles acarreta ganhos genéticos nos demais. A seleção para produção de raízes pode ser conduzida no caráter PT, pois sua avaliação é mais simples e correlaciona-se com a produção comercial, que é de maior importância. A seleção no caráter MV promove aumento nas características PMT e PMC e não causa efeitos nas demais. Para a maioria das características houve predominância dos efeitos ambientais sobre os genéticos. Os genótipos BD-15, BD-42 e BD-67 apresentaram melhor desempenho agronômico.

Palavras-chave: Ipomoea batatas L. (Lam.), produtividade, variabilidade genética, herdabilidade.

\section{ABSTRACT}

Agronomic performance and genetic parameters of sweet potato genotypes

The present study aimed to evaluate the agronomic performance of sweet potato genotypes and estimating genetic parameters. The experiment consisted of 65 varieties (clones) of the sweet potato germplasm bank from the Federal University of the Jequitinhonha and Mucuri Valley, installed in the design of randomized blocks with three replications and 15 plants per plot. Were evaluated green mass of branches (MV), total productivity of roots (PT), average weight of total roots (PMT), commercial yield (PC), the average weight of marketable roots (PMC), shape of roots (FORM) and insect resistance (IR). Considering the gains of selection for these characteristics, we infer that clone selection can lead to significant genetic progress. The characters PT, PMT, PC and PMC are positively correlated, so selecting any of them carries genetic gains in others. Selection for root production can be conducted in the character PT because its evaluation is simpler and correlates with the commercial production, which is of utmost importance. The selection promotes increases in MV character in PMC and PMT and causes no effects in others. For most traits were prevalent environmental effects on genetic. The BD-15, BD-42 and BD-67 genotypes showed better agronomic performance.

Keywords: Ipomoea batatas L. (Lam.), root yield, genetic variability, heritability.

(Recebido para publicação em 21 de janeiro de 2014; aceito em 4 de setembro de 2014) (Received on January 21, 2014; accepted on September 4, 2014)

\begin{abstract}
A batata-doce (Ipomoea batatas L. (Lam.)) é uma hortaliça tuberosa, rústica, de ampla adaptação, com alta tolerância à seca e de fácil produção (Cavalcante et al., 2006). Por ser uma cultura tradicional no Brasil, é bastante disseminada, cultivada principalmente por pequenos produtores rurais, com área plantada de 41.999 ha e rendimento médio de 11,84 t/ha (IBGE, 2010).

Devido aos teores de carboidratos, açúcares, sais minerais e vitaminas $\mathrm{A}$, C e do complexo B (Azevedo et al., 2002; Andrade \& Martins, 2002) é uma
\end{abstract}

boa alternativa como fonte de nutrientes e energia. Estes atributos, associados à sua versatilidade, possibilitam o uso da batata-doce para múltiplos fins. Na alimentação humana pode ser consumida assada, cozida, frita ou como farinha, enquanto que os animais podem se alimentar tanto de suas ramas quanto das raízes, frescas ou na forma de silagem (Andrade Júnior et al., 2009; Viana et al., 2011; Andrade Júnior et al., 2012; Figueiredo et al., 2012). Possui também grande potencial para extração de amido e para a produção de etanol (Oliveira et al., 2005; Andrade Júnior et al., 2009; Gonçalves Neto et al., 2011).

Pertencente à família Convolvulaceae, a batata-doce é a única espécie cujos indivíduos são hexaplóides $(2 \mathrm{n}=6 \mathrm{x}=$ 90), sendo este nível de ploidia provavelmente responsável pela alta variabilidade genética encontrada (Ritschel et al., 2010). Nas diversas regiões do Brasil onde é cultivada, observa-se uma grande diversidade genética, provavelmente decorrente das frequentes introduções de plantas desta espécie oriundas de locais distantes do centro de origem, 
diversificação ou cultivo, associadas à sua eficiente reprodução sexuada e assexuada (Silva et al., 2012). Esta variabilidade proporciona uma grande quantidade de genótipos a serem testados, a fim de se obter novas cultivares por meio da seleção e do melhoramento genético, o que segundo Cavalcante et al. (2003) constitui-se em um trabalho contínuo e dinâmico.

Cavalcante et al. (2009) relatam que ainda não há variedades específicas recomendadas para cultivo em cada região. Entretanto, a exploração da variabilidade genética existente nas diversas populações da espécie foi o foco de muitos trabalhos, possibilitando a seleção de genótipos para inúmeros propósitos. Entre estes propósitos destacam-se: resistência a pragas e doenças (Azevedo et al., 2002); melhor qualidade nutricional (Andrade Júnior et al., 2012; Azevedo et al., 2014); maior densidade de raízes (Cardoso et al., 2007); maior teor de matéria seca e produção de biomassa (Gonçalves Neto et al., 2012, Azevedo et al., 2014); maior aptidão para produção de etanol (Gonçalves Neto et al., 2011); maior produção de raízes para alimentação humana (Cardoso et al., 2005); e maior produção de ramas para alimentação animal (Viana et al., 2011; Andrade Júnior et al., 2012).

Para o melhoramento genético, as análises biométricas, sobretudo as estimativas de parâmetros genéticos como variância genotípica, coeficiente de variação genético, herdabilidade e índice de variação são determinantes na escolha de métodos de melhoramento mais adequados à cultura. Isto permite fazer inferências sobre a predição de ganhos com a seleção (Cruz et al., 2012). Já o estudo da correlação se destaca pelo conhecimento da influência de um caráter sobre outro, facilitando os trabalhos de seleção em caracteres de difícil avaliação, quando comparado a outro caráter associado.

Objetivou-se identificar genótipos promissores do banco de germoplasma de batata-doce da Universidade Federal dos Vales do Jequitinhonha e Mucuri e estimar parâmetros genéticos em genótipos de batata-doce.

\section{MATERIAL E MÉTODOS}

O experimento foi conduzido no Setor de Olericultura do Campus JK da UFVJM, município de Diamantina-MG (18 $12^{\circ} 01^{\prime \prime} \mathrm{S}, 43^{\circ} 34^{\prime} 20^{\prime \prime} \mathrm{O}$, altitude $1.387 \mathrm{~m}$ ), em Neossolo Quartzarênico Órtico Típico. No período de condução do experimento (dezembro de 2007 a abril de 2008) a precipitação média foi de $5,3 \mathrm{~mm}$ e a temperatura variou entre 11,0 e $30,4^{\circ} \mathrm{C}$.

As ramas para o plantio foram selecionadas, com três meses de antecedência, a partir do banco de germoplasma da UFVJM. Estas ramas foram multiplicadas em vasos com capacidade de cinco litros contendo substrato Plantmax Hortaliças sob estufa do tipo arco. Em dezembro de 2007 foi feito o plantio, quando foram utilizadas ramas com oito entrenós, ficando enterrados de três a quatro transversalmente sobre a leira, com 10 a $15 \mathrm{~cm}$ de profundidade. $\mathrm{O}$ delineamento experimental utilizado foi em blocos ao acaso com 65 tratamentos (genótipos) e três repetições, com parcelas de 4,5 m de comprimento, espaçadas entre si de $1 \mathrm{~m}$, onde cada parcela recebeu 15 plantas de forma equidistante. A área para o plantio foi preparada através de uma aração e uma gradagem com posterior sulcamento do terreno para a formação das leiras. As adubações de plantio, cobertura, irrigação e capina foram feitas de acordo com o recomendado para a cultura (Filgueira, 2008).

A colheita foi realizada cinco meses após o plantio, quando foram avaliadas características de produção e qualidade das raízes. A produtividade total de raízes e da massa verde de ramas foi obtida através de pesagem. A produtividade comercial de raízes foi calculada considerando-se aquelas com peso entre 100 e 800 gramas, sem rachaduras, não deformadas, não esverdeadas, não brocadas e sem veias.

O peso médio de todas as raízes foi obtido através da razão entre o peso total e o número total de raízes para cada parcela. O peso médio de raízes comerciais foi obtido através da razão entre o peso comercial e o número de raízes comerciais para cada parcela.

O formato da raiz foi avaliado atribuindo-se notas de 1 a 5 (Azevedo et al., 2002), sendo a nota $1=$ raiz com formato fusiforme, regular, sem veias ou qualquer tipo de rachaduras; 2 = raiz com formato considerado bom, próximo de fusiforme, com algumas veias; $3=$ raiz com formato desuniforme, com veias e bastante irregular; $4=$ raízes muito grandes, com veias e rachaduras, indesejáveis comercialmente; e 5= raízes totalmente fora dos padrões comerciais, muito irregulares e deformadas, com muitas veias e rachaduras.

A resistência a insetos de solo foi avaliada através de notas de 1 a 5 (Azevedo et al., 2002), sendo a nota $1=$ raízes livres de danos causados por insetos, com aspecto comercial desejável; 2= raízes com poucos danos, mas com presença de algumas galerias e furos nas raízes; 3 = raízes com danos bastante perceptíveis (presença de galeria e furos nas raízes em maior intensidade), com aspecto comercial prejudicado; 4= raízes com muitos danos, impróprias para comercialização (presença de muitas galerias, furos e início de apodrecimento); e 5= raízes inadequadas para fins comerciais (repletas de galerias, furos e apodrecimento mais avançado).

Os dados obtidos foram submetidos à análise de variância e as médias dos tratamentos comparadas pelo teste Scott-Knott, a 5\% de significância. A partir das esperanças dos quadrados médios das análises de variância, foram estimadas as herdabilidade no sentido amplo em nível de parcelas $\left(\mathrm{h}_{\mathrm{p}}^{2}\right)$ e em nível de média $\left(\mathrm{h}_{\mathrm{m}}^{2}\right)$. Também foram estimados os coeficientes de correlação genotípica utilizando-se o software genético-estatístico Genes (Cruz, 2013).

O ganho esperado com seleção (Gs) foi estimado baseando-se na média fenotípica dos 13 (20\%) melhores genótipos, onde $\mathrm{Gs}=\mathrm{ds} \times \mathrm{h}_{\mathrm{m}}{ }_{\mathrm{m}}$, sendo ds (diferencial de seleção) a diferença entre a média dos genótipos selecionados e a média geral, para cada caráter avaliado.

\section{RESULTADOS E DISCUSSÃO}

A característica resistência a insetos de solo não apresentou efeito significativo de genótipo pelo teste $F$, já para as outras características avaliadas verificaram-se diferenças significativas 
Tabela 1. Parâmetros genéticos e média da produtividade de massa verde das ramas (MV), produtividade total das raízes (PT), peso médio das raízes totais (PMT), produtividade de raízes comerciais (PC), produtividade média das raízes comerciais (PMC), formato de raízes (FORM) e resistência a insetos (RI) em clones de batata-doce (genetic parameters and fresh matter of branches (MV), total yield of roots (PT), average weight of total roots (PMT), marketable yield (PC), mean average yield of marketable roots (PMC), root format (FORM) and insect resistance (IR) of sweet potato clones). Diamantina, UFVJM, 2008.

\begin{tabular}{|c|c|c|c|c|c|c|c|}
\hline Genótipos & MV (t/ha) $)^{* * *}$ & PT (t/ha) ${ }^{* *}$ & PC (t/ha) $)^{* *}$ & PMT (g) & PMC (g) ${ }^{* * *}$ & FORM $^{* *}$ & $\mathbf{R I}^{\mathrm{ns}}$ \\
\hline Arruba & $1,74 \mathrm{~b}$ & $10,42 \mathrm{c}$ & $5,97 \mathrm{~b}$ & $95,88 \mathrm{~b}$ & $184,73 \mathrm{a}$ & $3,75 \mathrm{a}$ & 1,74 \\
\hline Bat. mandioca & 7,97 a & $4,14 \mathrm{c}$ & $2,29 \mathrm{~b}$ & $89,40 \mathrm{~b}$ & $153,33 \mathrm{~b}$ & $4,26 \mathrm{a}$ & 1,16 \\
\hline BD-01 & $1,58 \mathrm{~b}$ & $7,05 \mathrm{c}$ & $4,63 \mathrm{~b}$ & $92,75 \mathrm{~b}$ & $168,71 \mathrm{~b}$ & $4,08 \mathrm{a}$ & 1,48 \\
\hline BD-02 & $1,93 \mathrm{~b}$ & $9,18 \mathrm{c}$ & $5,92 \mathrm{~b}$ & $93,57 \mathrm{~b}$ & $145,61 \mathrm{~b}$ & $2,30 \mathrm{~b}$ & 1,22 \\
\hline BD-03 & $2,62 \mathrm{~b}$ & $8,63 \mathrm{c}$ & $3,35 \mathrm{~b}$ & $54,05 \mathrm{~b}$ & $132,98 \mathrm{~b}$ & $3,62 \mathrm{a}$ & 1,39 \\
\hline BD-04 & $3,28 \mathrm{~b}$ & $7,34 \mathrm{c}$ & $3,83 \mathrm{~b}$ & $72,42 \mathrm{~b}$ & $177,50 \mathrm{a}$ & $3,65 \mathrm{a}$ & 1,61 \\
\hline BD-05 & $3,36 \mathrm{~b}$ & $7,61 \mathrm{c}$ & $4,08 \mathrm{~b}$ & $67,78 \mathrm{~b}$ & $127,98 \mathrm{~b}$ & $3,93 \mathrm{a}$ & 1,51 \\
\hline BD-06 & $2,61 \mathrm{~b}$ & 17,84 a & 12,19 a & 125,30 a & 189,46 a & $2,22 \mathrm{~b}$ & 2,02 \\
\hline BD-07 & $1,32 \mathrm{~b}$ & $10,17 \mathrm{c}$ & $2,28 \mathrm{~b}$ & $63,36 \mathrm{~b}$ & $146,95 \mathrm{~b}$ & $2,78 \mathrm{~b}$ & 1,26 \\
\hline BD-08 & $0,56 \mathrm{~b}$ & $5,96 \mathrm{c}$ & $2,59 \mathrm{~b}$ & $58,50 \mathrm{~b}$ & $147,78 \mathrm{~b}$ & $2,98 \mathrm{~b}$ & 1,53 \\
\hline BD-10 & $1,23 \mathrm{~b}$ & $9,50 \mathrm{c}$ & $3,46 \mathrm{~b}$ & $58,11 \mathrm{~b}$ & $137,22 \mathrm{~b}$ & $3,08 \mathrm{a}$ & 1,54 \\
\hline BD-11 & $1,23 \mathrm{~b}$ & $12,75 \mathrm{~b}$ & $5,51 \mathrm{~b}$ & $93,39 \mathrm{~b}$ & $154,45 \mathrm{~b}$ & $2,12 \mathrm{~b}$ & 1,88 \\
\hline BD-13 & $3,92 \mathrm{~b}$ & $8,67 \mathrm{c}$ & $6,71 \mathrm{~b}$ & $97,90 \mathrm{~b}$ & $158,79 \mathrm{~b}$ & $3,05 \mathrm{a}$ & 1,28 \\
\hline BD-14 & $3,57 \mathrm{~b}$ & $10,59 \mathrm{c}$ & $5,86 \mathrm{~b}$ & $91,04 \mathrm{~b}$ & $157,94 \mathrm{~b}$ & $3,17 \mathrm{a}$ & 1,19 \\
\hline BD-15 & 4,94 a & 19,77 a & 13,92 a & 146,52 a & 199,76 a & $2,30 \mathrm{~b}$ & 1,93 \\
\hline BD-16 & $0,70 \mathrm{~b}$ & 13,96 b & $6,54 \mathrm{~b}$ & $75,32 \mathrm{~b}$ & $133,33 \mathrm{~b}$ & $3,23 \mathrm{a}$ & 1,65 \\
\hline BD-17 & 6,44 a & $9,15 \mathrm{c}$ & $6,51 \mathrm{~b}$ & 123,23 a & 201,72 a & $3,28 \mathrm{a}$ & 1,36 \\
\hline BD-20 & $1,98 \mathrm{~b}$ & $10,48 \mathrm{c}$ & $7,70 \mathrm{~b}$ & $88,41 \mathrm{~b}$ & $144,70 \mathrm{~b}$ & $2,90 \mathrm{~b}$ & 2,08 \\
\hline BD-21 & $4,64 \mathrm{a}$ & 13,47 b & $5,74 \mathrm{~b}$ & $77,49 \mathrm{~b}$ & $135,00 \mathrm{~b}$ & $2,95 \mathrm{~b}$ & 1,41 \\
\hline BD-22 & $2,28 \mathrm{~b}$ & $12,62 \mathrm{~b}$ & $7,27 \mathrm{~b}$ & $132,64 \mathrm{a}$ & 191,89 a & $3,35 \mathrm{a}$ & 1,71 \\
\hline BD-23 & 5,33 a & $9,13 \mathrm{c}$ & $6,04 \mathrm{~b}$ & $116,73 \mathrm{a}$ & 253,06 a & $3,44 \mathrm{a}$ & 1,45 \\
\hline BD-24 & $1,86 \mathrm{~b}$ & $14,54 \mathrm{~b}$ & $9,04 \mathrm{a}$ & $91,90 \mathrm{~b}$ & $167,80 \mathrm{~b}$ & $2,02 \mathrm{~b}$ & 1,66 \\
\hline BD-25 & $3,90 \mathrm{~b}$ & $10,63 \mathrm{c}$ & $5,78 \mathrm{~b}$ & 120,25 a & 211,11 a & $3,18 \mathrm{a}$ & 1,09 \\
\hline BD-25TO & $0,81 \mathrm{~b}$ & 19,32 a & 10,94 a & $73,11 \mathrm{~b}$ & $97,61 \mathrm{~b}$ & $1,75 \mathrm{~b}$ & 1,33 \\
\hline BD-26 & $3,17 \mathrm{~b}$ & 17,38 a & 13,04 a & 125,25 a & $176,33 \mathrm{a}$ & $2,12 \mathrm{~b}$ & 1,50 \\
\hline BD-28 & 8,14 a & $8,68 \mathrm{c}$ & $5,53 \mathrm{~b}$ & $107,68 \mathrm{~b}$ & $140,19 \mathrm{~b}$ & $2,90 \mathrm{~b}$ & 1,04 \\
\hline BD-29 & $0,73 \mathrm{~b}$ & $5,53 \mathrm{c}$ & $3,14 \mathrm{~b}$ & $61,76 b$ & $123,33 \mathrm{~b}$ & $4,15 \mathrm{a}$ & 1,87 \\
\hline BD-30 & $4,17 \mathrm{~b}$ & $8,69 \mathrm{c}$ & $3,71 \mathrm{~b}$ & $107,98 \mathrm{~b}$ & $148,00 \mathrm{~b}$ & $3,56 \mathrm{a}$ & 1,40 \\
\hline BD-31TO & $1,07 \mathrm{~b}$ & $8,90 \mathrm{c}$ & $3,62 \mathrm{~b}$ & $95,05 \mathrm{~b}$ & $165,37 \mathrm{~b}$ & $3,52 \mathrm{a}$ & 1,72 \\
\hline BD-32 & $3,69 \mathrm{~b}$ & $5,89 \mathrm{c}$ & $2,91 \mathrm{~b}$ & $70,64 \mathrm{~b}$ & $156,67 \mathrm{~b}$ & $3,80 \mathrm{a}$ & 1,37 \\
\hline BD-33 & $2,29 \mathrm{~b}$ & $5,81 \mathrm{c}$ & $3,27 \mathrm{~b}$ & $73,84 \mathrm{~b}$ & $125,60 \mathrm{~b}$ & $3,92 \mathrm{a}$ & 1,45 \\
\hline BD-34 & $2,55 \mathrm{~b}$ & $6,46 \mathrm{c}$ & $2,86 \mathrm{~b}$ & $53,53 \mathrm{~b}$ & $129,88 \mathrm{~b}$ & $4,23 \mathrm{a}$ & 1,46 \\
\hline BD-36 & $3,49 \mathrm{~b}$ & $5,80 \mathrm{c}$ & $3,78 \mathrm{~b}$ & 205,10 a & $126,43 \mathrm{~b}$ & $4,30 \mathrm{a}$ & 1,50 \\
\hline BD-38 & $3,67 \mathrm{~b}$ & 19,11 a & 13,70 a & 128,22 a & 204,45 a & $1,78 \mathrm{~b}$ & 1,78 \\
\hline BD-39 & $3,94 \mathrm{~b}$ & 17,72 a & 10,26 a & 186,88 a & $182,10 \mathrm{a}$ & $3,28 \mathrm{a}$ & 1,29 \\
\hline BD-42 & $6,01 \mathrm{a}$ & $12,32 \mathrm{~b}$ & 9,30 a & 129,81 a & 186,33 a & $2,17 \mathrm{~b}$ & 1,46 \\
\hline BD-43 & $2,22 \mathrm{~b}$ & $9,49 \mathrm{c}$ & $4,39 \mathrm{~b}$ & $79,85 \mathrm{~b}$ & $134,82 \mathrm{~b}$ & $3,87 \mathrm{a}$ & 1,41 \\
\hline BD-44 & $1,46 \mathrm{~b}$ & $12,39 \mathrm{~b}$ & $5,24 \mathrm{~b}$ & $54,32 \mathrm{~b}$ & $123,56 \mathrm{~b}$ & $3,67 \mathrm{a}$ & 1,52 \\
\hline BD-45 & $3,00 \mathrm{~b}$ & 16,82 a & 12,84 a & 121,34 a & $180,56 \mathrm{a}$ & $1,82 \mathrm{~b}$ & 2,45 \\
\hline BD-46 & $4,17 \mathrm{~b}$ & $7,29 \mathrm{c}$ & $3,57 \mathrm{~b}$ & $89,30 \mathrm{~b}$ & $163,48 \mathrm{~b}$ & $2,93 \mathrm{~b}$ & 1,34 \\
\hline BD-47 & $3,67 \mathrm{~b}$ & $9,89 \mathrm{c}$ & $4,91 \mathrm{~b}$ & $109,34 \mathrm{~b}$ & 187,50 a & $3,30 \mathrm{a}$ & 1,42 \\
\hline BD-48 & $3,65 \mathrm{~b}$ & $7,29 \mathrm{c}$ & $3,80 \mathrm{~b}$ & $94,20 \mathrm{~b}$ & $154,10 \mathrm{~b}$ & $4,21 \mathrm{a}$ & 1,47 \\
\hline BD-49 & $3,73 \mathrm{~b}$ & $8,00 \mathrm{c}$ & $4,07 \mathrm{~b}$ & $89,72 \mathrm{~b}$ & $181,39 \mathrm{a}$ & $3,67 \mathrm{a}$ & 1,75 \\
\hline BD-50 & $2,67 \mathrm{~b}$ & $7,37 \mathrm{c}$ & $3,93 \mathrm{~b}$ & $94,07 \mathrm{~b}$ & 231,15 a & $3,70 \mathrm{a}$ & 1,29 \\
\hline
\end{tabular}


Tabela 1. Continuação

\begin{tabular}{|c|c|c|c|c|c|c|c|}
\hline Genótipos & MV (t/ha) $)^{* *}$ & PT (t/ha) $)^{* * k}$ & PC (t/ha) $)^{* *}$ & $\operatorname{PMT}(\mathrm{g})^{* *}$ & $\operatorname{PMC}(g)^{* *}$ & FORM $^{* * *}$ & $\mathbf{R I}^{\mathrm{ns}}$ \\
\hline Arruba & $1,74 \mathrm{~b}$ & $10,42 \mathrm{c}$ & $5,97 \mathrm{~b}$ & $95,88 \mathrm{~b}$ & $184,73 \mathrm{a}$ & $3,75 \mathrm{a}$ & 1,74 \\
\hline Bat. mandioca & $7,97 \mathrm{a}$ & $4,14 \mathrm{c}$ & $2,29 \mathrm{~b}$ & $89,40 \mathrm{~b}$ & $153,33 \mathrm{~b}$ & $4,26 \mathrm{a}$ & 1,16 \\
\hline BD-34 & $2,55 \mathrm{~b}$ & $6,46 \mathrm{c}$ & $2,86 \mathrm{~b}$ & $53,53 \mathrm{~b}$ & $129,88 \mathrm{~b}$ & $4,23 \mathrm{a}$ & 1,46 \\
\hline BD-36 & $3,49 \mathrm{~b}$ & $5,80 \mathrm{c}$ & $3,78 \mathrm{~b}$ & 205,10 a & $126,43 \mathrm{~b}$ & $4,30 \mathrm{a}$ & 1,50 \\
\hline BD-38 & $3,67 \mathrm{~b}$ & 19,11 a & 13,70 a & 128,22 a & 204,45 a & $1,78 \mathrm{~b}$ & 1,78 \\
\hline BD-39 & $3,94 \mathrm{~b}$ & 17,72 a & 10,26 a & 186,88 a & $182,10 \mathrm{a}$ & $3,28 \mathrm{a}$ & 1,29 \\
\hline BD-42 & $6,01 \mathrm{a}$ & $12,32 \mathrm{~b}$ & 9,30 a & 129,81 a & 186,33 a & $2,17 \mathrm{~b}$ & 1,46 \\
\hline BD-43 & $2,22 \mathrm{~b}$ & $9,49 \mathrm{c}$ & $4,39 \mathrm{~b}$ & $79,85 \mathrm{~b}$ & $134,82 \mathrm{~b}$ & $3,87 \mathrm{a}$ & 1,41 \\
\hline BD-44 & $1,46 \mathrm{~b}$ & $12,39 \mathrm{~b}$ & $5,24 \mathrm{~b}$ & $54,32 \mathrm{~b}$ & $123,56 \mathrm{~b}$ & $3,67 \mathrm{a}$ & 1,52 \\
\hline BD-45 & $3,00 \mathrm{~b}$ & 16,82 a & 12,84 a & 121,34 a & $180,56 \mathrm{a}$ & $1,82 \mathrm{~b}$ & 2,45 \\
\hline BD-46 & $4,17 \mathrm{~b}$ & $7,29 \mathrm{c}$ & $3,57 \mathrm{~b}$ & $89,30 \mathrm{~b}$ & $163,48 \mathrm{~b}$ & $2,93 \mathrm{~b}$ & 1,34 \\
\hline BD-47 & $3,67 \mathrm{~b}$ & $9,89 \mathrm{c}$ & $4,91 \mathrm{~b}$ & $109,34 \mathrm{~b}$ & 187,50 a & $3,30 \mathrm{a}$ & 1,42 \\
\hline BD-48 & $3,65 \mathrm{~b}$ & $7,29 \mathrm{c}$ & $3,80 \mathrm{~b}$ & $94,20 \mathrm{~b}$ & $154,10 \mathrm{~b}$ & $4,21 \mathrm{a}$ & 1,47 \\
\hline BD-49 & $3,73 \mathrm{~b}$ & $8,00 \mathrm{c}$ & $4,07 \mathrm{~b}$ & $89,72 \mathrm{~b}$ & $181,39 \mathrm{a}$ & $3,67 \mathrm{a}$ & 1,75 \\
\hline BD-50 & $2,67 \mathrm{~b}$ & $7,37 \mathrm{c}$ & $3,93 \mathrm{~b}$ & $94,07 \mathrm{~b}$ & 231,15 a & $3,70 \mathrm{a}$ & 1,29 \\
\hline BD-51 & $4,00 \mathrm{~b}$ & $5,20 \mathrm{c}$ & $2,52 \mathrm{~b}$ & $56,55 \mathrm{~b}$ & $135,00 \mathrm{~b}$ & $3,57 \mathrm{a}$ & 1,55 \\
\hline BD-52 & $3,23 \mathrm{~b}$ & $8,57 \mathrm{c}$ & $4,46 \mathrm{~b}$ & $94,72 \mathrm{~b}$ & $167,86 \mathrm{~b}$ & $3,17 \mathrm{a}$ & 1,35 \\
\hline BD-53 & $4,26 \mathrm{~b}$ & $11,07 \mathrm{c}$ & $5,17 \mathrm{~b}$ & $84,59 \mathrm{~b}$ & $182,48 \mathrm{a}$ & $3,73 \mathrm{a}$ & 1,44 \\
\hline BD-54 & $2,41 \mathrm{~b}$ & $9,10 \mathrm{c}$ & $4,65 \mathrm{~b}$ & $84,69 \mathrm{~b}$ & $144,89 \mathrm{~b}$ & $2,17 \mathrm{~b}$ & 1,43 \\
\hline BD-56 & $2,44 \mathrm{~b}$ & $9,02 \mathrm{c}$ & $5,63 \mathrm{~b}$ & $80,52 \mathrm{~b}$ & $149,36 \mathrm{~b}$ & $2,80 \mathrm{~b}$ & 1,42 \\
\hline BD-58 & $2,75 \mathrm{~b}$ & $7,79 \mathrm{c}$ & $3,23 \mathrm{~b}$ & $87,54 \mathrm{~b}$ & $149,44 \mathrm{~b}$ & $4,02 \mathrm{a}$ & 1,40 \\
\hline BD-65 & $3,41 \mathrm{~b}$ & $12,51 \mathrm{~b}$ & 9,91 a & 139,49 a & $158,19 \mathrm{~b}$ & $1,95 \mathrm{~b}$ & 1,22 \\
\hline BD-66 & $3,20 \mathrm{~b}$ & $12,47 \mathrm{~b}$ & $7,54 \mathrm{~b}$ & $104,45 \mathrm{~b}$ & 186,24 a & $2,33 \mathrm{~b}$ & 1,37 \\
\hline BD-67 & 6,69 a & $12,81 \mathrm{~b}$ & 9,52 a & $103,90 \mathrm{~b}$ & $157,32 \mathrm{~b}$ & $1,60 \mathrm{~b}$ & 1,57 \\
\hline BD-113TO & $1,30 \mathrm{~b}$ & $11,15 \mathrm{c}$ & $4,50 \mathrm{~b}$ & $96,06 \mathrm{~b}$ & $147,82 \mathrm{~b}$ & $2,15 \mathrm{~b}$ & 1,36 \\
\hline Braz. Rosada & $3,21 \mathrm{~b}$ & $13,00 \mathrm{~b}$ & 10,20 a & $155,24 \mathrm{a}$ & 250,71 a & $3,23 \mathrm{a}$ & 1,61 \\
\hline Braz. Roxa & 5,18 a & $11,24 \mathrm{c}$ & $6,84 \mathrm{~b}$ & $86,27 \mathrm{~b}$ & $147,70 \mathrm{~b}$ & $2,72 \mathrm{~b}$ & 1,22 \\
\hline Cambraia & $2,47 \mathrm{~b}$ & $10,17 \mathrm{c}$ & $5,69 \mathrm{~b}$ & $95,94 \mathrm{~b}$ & $159,52 \mathrm{~b}$ & $2,63 \mathrm{~b}$ & 1,68 \\
\hline Coquinho & $2,03 \mathrm{~b}$ & $7,45 \mathrm{c}$ & $2,69 \mathrm{~b}$ & $67,09 \mathrm{~b}$ & $121,67 \mathrm{~b}$ & $3,37 \mathrm{a}$ & 1,61 \\
\hline Cor. Magoado & $2,09 \mathrm{~b}$ & $8,27 \mathrm{c}$ & $3,60 \mathrm{~b}$ & $73,21 \mathrm{~b}$ & $172,22 \mathrm{a}$ & $4,18 \mathrm{a}$ & 1,70 \\
\hline Espanhola & $4,58 \mathrm{a}$ & $5,07 \mathrm{c}$ & $2,23 \mathrm{~b}$ & $54,56 \mathrm{~b}$ & $116,89 \mathrm{~b}$ & $4,30 \mathrm{a}$ & 1,43 \\
\hline Licuri & $1,20 \mathrm{~b}$ & $8,86 \mathrm{c}$ & $5,62 \mathrm{~b}$ & $76,47 \mathrm{~b}$ & $133,27 \mathrm{~b}$ & $3,48 \mathrm{a}$ & 1,75 \\
\hline Marmel & $1,67 \mathrm{~b}$ & $3,71 \mathrm{c}$ & $3,09 \mathrm{~b}$ & $72,10 \mathrm{~b}$ & $179,26 \mathrm{a}$ & $4,22 \mathrm{a}$ & 1,76 \\
\hline Palmas & $7,74 \mathrm{a}$ & $10,56 \mathrm{c}$ & $6,56 \mathrm{~b}$ & $97,41 \mathrm{~b}$ & $135,42 \mathrm{~b}$ & $3,90 \mathrm{a}$ & 1,26 \\
\hline Princesa & $3,22 \mathrm{~b}$ & 15,99 a & $11,71 \mathrm{a}$ & $103,52 \mathrm{~b}$ & 242,58 a & $2,63 \mathrm{~b}$ & 1,85 \\
\hline Tomba Carro & $3,84 \mathrm{~b}$ & $13,83 \mathrm{~b}$ & $9,68 \mathrm{a}$ & $106,55 \mathrm{~b}$ & $165,67 \mathrm{~b}$ & $2,77 \mathrm{~b}$ & 1,60 \\
\hline $\mathrm{CV}_{\mathrm{e}}(\%)$ & 61,61 & 34,85 & 41,6 & 39,91 & 25,73 & 23 & 24,33 \\
\hline $\mathrm{CV}_{\mathrm{g}}^{\mathrm{e}}(\%)$ & 38,79 & 31,14 & 45,83 & 21,35 & 13,16 & 19,98 & 8,59 \\
\hline $\mathrm{b}$ & 0,62 & 0,89 & 1,08 & 0,53 & 0,51 & 0,86 & 0,33 \\
\hline$M_{65}$ & 3,20 & 10,33 & 6,02 & 95,35 & 162,22 & 3,16 & 1,52 \\
\hline $\mathrm{M}_{13}$ & 5,85 & 16,37 & 11,32 & 141,48 & 210,46 & 2,01 & 1,22 \\
\hline$d_{s}$ & 2,65 & 6,03 & 5,31 & 46,13 & 48,24 & $-1,14$ & $-0,30$ \\
\hline $\mathrm{h}_{\mathrm{m}}^{\mathrm{s}}$ & 0,53 & 0,71 & 0,78 & 0,46 & 0,44 & 0,69 & 0,25 \\
\hline $\mathrm{G}_{\mathrm{s}}^{\mathrm{m}}$ & 1,42 & 4,26 & 4,13 & 21,31 & 20,98 & $-0,79$ & $-0,07$ \\
\hline$P_{m}^{s}$ & 4,62 & 14,59 & 10,15 & 116,66 & 183,20 & 2,37 & 1,44 \\
\hline $\mathrm{G}_{\mathrm{s}}(\%)$ & 44,20 & 41,20 & 68,70 & 22,40 & 12,90 & $-25,00$ & $-4,90$ \\
\hline
\end{tabular}

NS não significativo, *significativo a $5 \%, * *$ significativo a $1 \%$ de probabilidade pelo teste F. Médias seguidas pela mesma letra não diferem entre si pelo teste de Scott-Knott a 5\% de probabilidade. Valores em negrito se referem aos valores dos genótipos selecionados. CV: coeficientes de variação ambiental; $\mathrm{CV}_{\mathrm{g}}$ : coeficientes de variação genético; $\mathrm{h}^{2}$ : herdabilidades em nível da média; $\mathrm{M}_{65}:$ médias geral; $\mathrm{M}_{13}: \mathrm{Média}$ dos 13 $(20 \%)$ genótipos selecionados; $d$ : diferencial de seleção; $\mathrm{G}$ : ganho genético esperado com a seleção e $\mathrm{P}$ : média esperada para a população melhorada ( ${ }^{\mathrm{NS}}$ not significant at $5 \%$, *significant at $5 \%$ and ** significant at $1 \%$ probability by $\mathrm{F}$ test. Means followed by the same letter do not differ by the Scott-Knott test at 5\% probability. Values in bold refer to the selected genotypes. $\mathrm{CV}_{\mathrm{e}}$ : coefficients of environmental variation; $\mathrm{CV}_{\mathrm{g}}$ : coefficients of genetic variation; $\mathrm{h}_{\mathrm{m}}^{2}$ : heritability level on average; $\mathrm{M}_{65}$ : general average; $\mathrm{M}_{13}: \mathrm{Mean}_{\text {of }} 13$ (20\%) selected genotypes; ds: selection differential; Gs: genetic gain expected from the selection and Pm: expected average for the improved population). 
Tabela 2. Coeficientes de correlação genotípico entre 7 características avaliadas em 65 clones de batata-doce (genotypic correlation coefficients among seven traits evaluated in 65 clones of sweet potato). Diamantina, UFVJM, 2008.

\begin{tabular}{lccccc}
\hline Caracteres $^{1}$ & PT (t/ha) & PMT $(\mathbf{g})$ & PC $(\mathbf{t} / \mathbf{h a})$ & PMC $(\mathbf{g})$ & FORM \\
\hline MV (t/ha) & $-0,09^{\text {ns }}$ & $0,61^{* *}$ & $0,16^{\mathrm{ns}}$ & $0,36^{* *}$ & $-0,01^{\mathrm{ns}}$ \\
PT (t/ha) & - & $0,59^{* *}$ & $0,99^{* *}$ & $0,38^{* *}$ & $-0,90^{* *}$ \\
PMT (g) & - & - & $0,75^{* *}$ & $0,67^{* *}$ & $-0,37^{* *}$ \\
PC (t/ha) & - & - & - & $0,59^{* *}$ & $-0,85^{* *}$ \\
PMC (g) & - & - & - & - & $-0,34^{* *}$ \\
\hline
\end{tabular}

${ }^{1}$ Caracteres: produtividade de massa verde das ramas (MV), produtividade total das raízes (PT), peso médio das raízes totais (PMT), produtividade de raízes comerciais (PC), peso médio das raízes comerciais (PMC) e formato das raízes (FORM) em clones de batata-doce. ${ }^{\mathrm{NS}}$ não significativo a $5 \%, *$ significativo a $5 \%,{ }^{*}$ significativo a $1 \%$ de probabilidade pelo teste $\mathrm{t}\left({ }^{1}\right.$ variables: fresh matter of branches (MV), total yield of roots (PT), average weight of total roots (PMT), marketable yield (PC), average weight of marketable roots (PMC) and shape of roots (FORM) in clones of sweet potato. ${ }^{\mathrm{NS}}$ not significant at $5 \%$, * significant at $5 \%$ and $* *$ significant at $1 \%$ probability by $\mathrm{t}$ test).

ao nível de $1 \%$ de significância.

Observa-se que os coeficientes de variação experimental $\left(\mathrm{CV}_{\mathrm{e}}\right)$ estão acima de $20 \%$ para todas as variáveis. A suscetibilidade destas variáveis aos efeitos do ambiente tem sido observada com frequência (Cavalcante et al., 2003; Cardoso et al., 2005; Cavalcante et al., 2006; Andrade Júnior et al., 2009; Moreira et al., 2011). Para as variáveis relacionadas à produção de raízes, uma das possíveis causas dos altos $\mathrm{CV}_{\mathrm{e}}$ é o fato destas estarem no subsolo, não sendo imediatamente visíveis. Este fato implica na colheita incompleta de algumas parcelas.

Embora os $\mathrm{CV}_{\mathrm{e}}$ tenham sido altos, as estimativas dos coeficientes de variação genético $\left(\mathrm{CV}_{\mathrm{g}}\right)$, das herdabilidades e do índice de variação $\left(\mathrm{CV}_{\mathrm{g}} / \mathrm{CV}_{\mathrm{e}}\right)$, representado por b (Tabela 1 ), foram bastante promissoras, exceto para o caráter resistência a insetos. Como estes últimos parâmetros medem o grau de determinação genético de um caráter, pode-se inferir que, a despeito da expressiva contribuição do ambiente para os caracteres em apreço, os clones em questão apresentam alta variabilidade genética.

As maiores estimativas de herdabilidade foram encontradas para produtividade total de raízes, produtividade de raízes comerciais e formato de raízes (Tabela 1), corroborando os resultados obtidos por Gonçalves Neto et al. (2012). Contudo, estes autores encontraram estimativas de herdabilidade elevadas $(95,90 \%)$ para a produtividade de massa verde de ramas, o que não foi verificado no presente trabalho.
A herdabilidade está diretamente envolvida na estimação do ganho genético com seleção, seja em nível de indivíduo, de parcela ou de média. Segundo Ivoglo et al. (2008), tal parâmetro representa a confiabilidade com a qual o valor fenotípico representa o valor genotípico, consequentemente, características com alta $\mathrm{h}^{2}$ implicam em menor influência do ambiente, o que aumenta o poder discriminatório dos mesmos e o ganho esperado com seleção. No caso da batata-doce, a herdabilidade no sentido amplo é importante devido aos efeitos de dominância e epistasia serem mantidos pela propagação vegetativa (Gonçalves Neto et al., 2012). Como este sistema de propagação é altamente eficiente para a espécie, estes efeitos são todos capitalizados nas variedades selecionadas para plantios comerciais.

Observa-se que a produtividade de raízes comerciais, único caráter com índice de variação superior a um, apresentou também as maiores herdabilidades e maior $\mathrm{CV}_{\mathrm{g}}$ (Tabela 1). Isto já é esperado, uma vez que a variância genética está presente no numerador do estimador de todos estes parâmetros.

Para a produtividade de massa verde de ramas, foram encontrados valores variando de 0,56 a $8,14 \mathrm{t} / \mathrm{ha}$, correspondente aos genótipos BD-08 e BD-28 respectivamente (Tabela 1). Este intervalo foi maior que o encontrado por Cavalcante et al. (2003) em Rio Largo-AL, Cavalcante et al. (2009, 2010) ambos em Junqueiro-AL que encontraram valores de 0,92 a 5,83 t/ha. Esta diferença pode ser explicada pelos efeitos ambientais e genéticos. Quanto aos efeitos ambientais, estes experimentos foram conduzidos em locais de menor altitude, maior temperatura e em solos com diferentes classificações, quando comparado a este experimento. Quanto aos efeitos genéticos, foram avaliados diferentes genótipos. Destacaram-se para esta característica os genótipos Batata mandioca, BD-15, BD-17, BD21, BD-23, BD-28, BD-42, BD-67, Brazlândia Roxa, Espanhola e Palmas. Além destes genótipos, fizeram parte dos 13 genótipos superiores os genótipos BD-30 e BD-53. Com a seleção destes genótipos é esperado um ganho de seleção de 44,2\% correspondendo à média esperada de 4,62 t/ha para a população melhorada.

Para a produtividade total de raízes, destacaram-se os genótipos BD-06, BD15, BD-25TO, BD-26, BD-38, BD-39, BD-45 e Princesa. Todos estes genótipos, com exceção do genótipo Princesa, apresentaram médias superiores à maior média encontrada por Moreira et al. (2011) para o genótipo ESAM 2, que se destacou como mais produtivo $(16,80 \mathrm{t} /$ ha). Poucas alterações foram observadas entre o grupo de genótipos que apresentaram maior produtividade total de raízes e maior produtividade comercial de raízes. Com maior produtividade comercial de raízes destacaram-se os genótipos BD-06, BD-15, BD-24, BD-25TO, BD-26, BD-38, BD-39, BD-42, BD-45, BD-65, BD-67, Brazlândia Rosada, Princesa e Tomba Carro. Dentre estes genótipos, seis (BD-06, BD-15, BD-26, BD-38, BD-45 e Princesa) se sobressaíram em relação aos melhores genótipos avaliados por Cavalcante et 
al. (2009) e Moreira et al. (2011) que encontraram 12,08 e 11,16 t/ha, respectivamente. A seleção dos 13 genótipos superiores para esta característica (BD06, BD-15, BD-25TO, BD-26, BD-38, BD-39, BD-42, BD-45, BD-65, BD-67, Brazlândia Rosada, Princesa e Tomba Carro) possibilitará o ganho de seleção de $68,7 \%$, sendo este o maior ganho de seleção predito entre as características avaliadas.

Para o peso médio de raízes totais destacaram-se os genótipos BD-06, BD-15, BD-17, BD-22, BD-23, BD-25, BD-26, BD-36, BD-38, BD-39, BD-42, BD-45, BD-65 e Brazlândia Rosada. O genótipo que apresentou maior média para esta característica foi o BD-36 com 205,10 g, aproximando-se da maior média verificada por Andrade Júnior et al. (2009) para esta mesma característica (293,02 g). Para o peso médio das raízes comerciais, foi encontrada uma grande variação das médias (97,61 a 253,06 g), sendo tal intervalo muito superior aos verificados por Andrade Júnior et al. (2009) que encontraram valores variando de 184,94 a 320,95 g. Os genótipos BD-06, BD-15, BD-17, BD-22, BD-23, BD-25, BD-38, BD-42, BD-47, BD-50, BD-66, Brazlândia Rosada e Princesa estão entre os genótipos superiores, e sua seleção possibilitará o ganho de seleção predito de $12,9 \%$, o que seria importante, uma vez que tal característica se correlaciona com a produtividade comercial de raízes (Tabela 1).

Quanto ao formato de raízes, foram encontrados valores variando de 1,16 a 4,30, aproximando do intervalo verificado por Gonçalves Neto et al. (2011) e Gonçalves Neto et al. (2012) que encontraram valores variando de 1,5 a 5,0 sendo contudo inferior ao intervalo encontrado por Andrade Júnior et al. (2012), que verificaram valores de 1,8 a 2,7. Os genótipos BD-02, BD-06, BD11, BD-24, BD-25TO, BD-26, BD-38, BD-42, BD-45, BD-54, BD-65, BD-67 e BD-113TO foram aqueles com melhor formato de raízes, do ponto de vista comercial, sendo que a seleção destes genótipos permitirá um ganho de seleção de $-0,79$, o que corresponde a $-25 \%$.

Para a característica resistência a insetos de solo foram encontrados valores variando de 1,04 a 2,45; no en- tanto não foi observada diferença entre os genótipos, indicando a ausência de variabilidade genética para esta característica, consequentemente, a seleção dos melhores genótipos corresponderia ao menor ganho de seleção entre as características avaliadas (-4,9\%).

As estimativas de herdabilidade no sentido amplo para estas características, exceto RI, permitem a obtenção de progressos genéticos significativos a partir da seleção de clones.

Pelas magnitudes das correlações genotípicas apresentadas na Tabela 2 , pode-se inferir que a produtividade de massa verde de ramas não apresenta influência no formato das raízes e na produtividade total e comercial de raízes, confirmando os resultados obtidos por Cavalcante et al. (2006) e Gonçalves Neto et al. (2012). Além disso, a produtividade de massa verde de ramas apresenta correlação positiva e significativa com o peso médio das raízes totais e peso médio de raízes comerciais (Tabela 2). Portanto, a seleção para massa verde, caráter importante para consumo animal na forma natural ou de silagem, pode não ter qualquer implicação na produtividade total e no formato da raiz, mas tende a aumentar seus pesos médios comerciais e totais. Em relação a estes dois últimos caracteres pode-se inferir que plantas com ramas maiores tendem a ter raízes também maiores.

Correlações positivas significativas também foram encontradas entre as características produtividade total e comercial de raízes, peso médio de raízes comerciais e totais. Quanto ao formato das raízes, verificaram-se correlações negativas e significativas com todas as demais características exceto a matéria verde (Tabela 2). Não é surpreendente a alta correlação observada entre o peso comercial e o formato uma vez que o critério de escolha das raízes comerciais leva à seleção de raízes com notas menores. Entretanto, a alta correlação do formato com o peso total é promissora, pois implica que a seleção de clones com maior produtividade leva à melhoria também do formato das raízes.

Verifica-se também que dentre os caracteres PT, PMT, PC, e PMC a seleção em qualquer um acarreta aumento nos demais. A seleção nos caracteres PT,
PMT, PC, e PMC ocasiona melhoria no caráter FORM. A seleção para produção de raízes pode ser conduzida no caráter PT, já que este caráter é mais simples de ser avaliado e é altamente correlacionado ao PC, que tem maior importância comercial. A seleção no caráter MV promove aumento nas características PMT e PMC e não causa efeitos nas demais. Para a maioria das características houve predominância dos efeitos ambientais sobre os genéticos. Os genótipos BD-15, BD-42 e BD-67 se destacaram por apresentar maiores valores de produtividade comercial de raízes e de matéria verde de ramas, apresentando potencial para alimentação humana e animal.

\section{AGRADECIMENTOS}

À FAPEMIG, ao CNPq e à CAPES pela concessão de bolsas de estudos e recursos financeiros para o desenvolvimento do projeto.

\section{REFERÊNCIAS}

ANDRADE JÚNIOR VC; VIANA DJS; FERNANDES JSC; FIGUEIREDO JA; NUNES UR; NEIVA IP. 2009. Selection of sweet potato clones for the region Alto Vale do Jequitinhonha. Horticultura Brasileira 27: 389-393.

ANDRADE JÚNIOR VC; VIANA DJS; PINTO NAVD; RIBEIRO KG; PEREIRA RC; NEIVA IP; AZEVEDO AM; ANDRADE PCR. 2012. Características produtivas e qualitativas de ramas e raízes de batata-doce. Horticultura Brasileira 30: 584-589.

ANDRADE RLP; MARTINS JFP. 2002. Influência da adição da fécula de batata-doce (Ipomoea batatas) sobre a viscosidade do permeado de soro de queijo. Ciência e Tecnologia de Alimentos 22: 249-253.

AZEVEDO AM; ANDRADE JÚNIOR VC; VIANA DJS; ELSAYED AYAM; PEDROSA CE; NEIVA IP; FIGUEIREDO JA. 2014. Influence of harvest time and cultivation sites on the productivity and quality of sweet potato. Horticultura Brasileira 32: 21-27.

AZEVEDO SM; MALUF WR; SILVEIRA MA; FREITAS JA. 2002. Reação de clones de batata-doce aos insetos de solo. Ciência e Agrotecnologia 26: 545-549.

CARDOSO AD; VIANA AES; MATSUMOTO SN; BONFIM NETO H; KHOURI CR; MELO TL. 2007. Características físicas e sensoriais de clones de batata-doce. Ciência e Agrotecnologia 31: 1760-1765.

CARDOSO AD; VIANA AES; RAMOS PAS; MATSUMOTO SN; AMARAL CLF; SEDIYAMA T; MORAIS OM. 2005. 
Avaliação de clones de batata-doce em Vitória da Conquista. Horticultura Brasileira 23: 911-914.

CAVALCANTE, M; FERREIRA PV; PAIXÃO SL; COSTA JC; PEREIRA RG; MADALENA JAS. 2009. Potenciais produtivo e genético de clones de batata-doce. Acta Scientiarum Agronomy 31: 421-426.

CAVALCANTE M; FERREIRA PV; PAIXÃO SL; COSTA JC; PEREIRA RG; MADALENA JA. 2010. Desempenho agronômico, dissimilaridade genética e seleção de genitores de batata doce para hibridização. Revista Brasileira de Ciências Agrárias 05: 485-490.

CAVALCANTE JT; FERREIRA PV; SOARES L. 2003. Avaliação de clones de batata-doce (Ipomoea batatas) em Rio Largo - Alagoas. Magistra 15: 13-17.

CAVALCANTE JT; FERREIRA PV; SOARES L; BORGES V; SILVA PP; SILVA JW. 2006. Análise de trilha em caracteres de rendimento de clones de batata-doce (Ipomoea batatas). Acta Scientiarum Agronomy 28: 261-266.

CRUZ CD. 2013. GENES - a software package for analysis in experimental statistics and quantitative genetics. Acta Scientiarum. 35: 271-276.

CRUZ CD; REGAZZI AJ; CARNEIRO PCS. 2012. Modelos biométricos aplicados ao melhoramento genético. 3. ed. Viçosa: UFV. 480p.
FILGUEIRA FAR. 2008. Novo manual de olericultura: Agrotecnologia moderna na produção e comercialização de hortaliças. Viçosa: UFV. 402p.

FIGUEIREDO JA; ANDRADE JÚNIOR VC; PEREIRA RC; RIBEIRO KG; VIANA DJS; NEIVA IP. 2012. Avaliação de silagens de ramas de batata-doce. Horticultura Brasileira 30: 708-712.

GONÇALVES NETO AC; MALUF WR; GOMES LAA; GONÇALVES RJS; SILVA VF; LASMAR A. 2011. Aptidões de genótipos de batata-doce para consumo humano, produção de etanol e alimentação animal. Pesquisa Agropecuária Brasileira 46: 1513-1520.

GONÇALVES NETO AC; MALUF WR; GOMES LAA; MACIEL GM; FERREIRA RPD; CARVALHO RC. 2012. Correlação entre caracteres e estimação de parâmetros populacionais para batata-doce. Horticultura Brasileira 30: 713-719.

IBGE. 2010. Produção Agrícola Municipal: Culturas Temporárias e Permanentes. 2012, 29 janeiro. Rio de Janeiro, v. 37, p.1-91. Disponível em: http://www.ibge.gov.br

IVOGLO MG; FAZUOLI LC; OLIVEIRAACB; GALLO PB; MISTRO JC; SILVAROLLAMB; TOMA-BRAGHINI M. 2008. Divergência genética entre progênies de café robusta. Bragantia 67: 823-83.

MOREIRA JN; QUEIROGA RC; SOUSA
JÚNIORAJL; SANTOS MA. 2011. Caracteres morfofisiológicos e produtivos de cultivares de batata-doce, em Mossoró-RN. Revista Verde 6: 161-167.

OLIVEIRAAP; OLVEIRA MRT; BARBOSA JA; SILVA GG; NOGUEIRA DH; MOURA MF; BRAZ MSS. 2005. Rendimento e qualidade de raízes de batata-doce adubada com níveis de uréia. Horticultura Brasileira 23: 925-928.

RITSCHEL PS; LOPES CA; HUAMÁN Z; FERREIRA ME; FRANCA FJ; MENÊZES JE; TEIXEIRA DMC; TORRES AC; CHARCHAR JM; THOMAZELLI L. 2010, 31 de janeiro. Organização do banco ativo de germoplasma de batata-doce: situação atual e perspectivas. In: QUEIROZ MA; GOEDERT CO; RAMOS SRR. (eds). Recursos genéticos e melhoramento de plantas para o Nordeste brasileiro: versão 1.0. Disponível em: http:// www.cpatsa.embrapa.br/ catalogo/livrorg/ batatadoce.pdf.

SILVA GO; PONIJALEKI R; SUINAGA FA. 2012. Divergência genética entre acessos de batata-doce utilizando caracteres fenotípicos de raiz. Horticultura Brasileira 30: 595-599.

VIANADJS; ANDRADE JUNIOR VC; RIBEIRO GK; PINTO NAVD; NEIVA IP; FIGUEIREDO JA; LEMOS VT; PEDROSA CE; AZEVEDO AM. 2011. Potencial de silagens de ramas de batata-doce para alimentação animal. Ciência Rural 41: 1466-1471. 